\title{
Local Stabilization for Discrete-Time Systems with Distributed State Delay and Fast-Varying Input Delay under Actuator Saturations
}

\author{
Yonggang Chen and Zidong Wang
}

\begin{abstract}
This paper is concerned with the local stabilization problem for discrete-time systems with both distributed state delay and fastvarying input delay under actuator saturations. By introducing some terms concerning the distributedly delayed state and the current state, a novel polytopic model is first proposed to characterize the delayed saturation nonlinearity. Then, by incorporating a piecewise Lyapunov functional and some summation inequalities, a sufficient condition is established by means of linear matrix inequalities under which the closedloop system is locally exponentially stable. Moreover, the conditions for two special cases with single state delay and single input delay are proposed. Subsequently, certain optimization problems are formulated with aim to maximize the estimate of the region of attraction. Finally, two examples show the effectiveness and values of the obtained results.
\end{abstract}

Index Terms-Local stabilization, discrete-time systems, distributed state delay, fast-varying input delay, actuator saturations.

\section{INTRODUCTION}

Time delays are often encountered in many real-world control systems and their existence is likely to lead to performance degradation or even instability of a control system. As such, the analysis and synthesis for time-delay systems have gained significant attention over the past three decades, see. e.g, [4], [12], [22], [24]. Overall, three types of time delays have been addressed in the literature, namely, discrete delays, distributed delays and neutral delays. In the context of linear matrix inequalities (LMIs), many advanced techniques have been developed for the convenience of controller/fiter design based on the utilization of some inequalities including Wirtinger-based inequalities [13], [14], free-matrix-based inequalities [25], [26], BesselLegendre inequalities [29] and reciprocally convex inequalities [15], [27]. However, it is noted that most available results have been concerned with a single discrete delay. For distributed delay systems, some recent results can be found in [3], [10], [11], [18].

Due to unavoidable physical constraints, actuator saturations are commonly encountered in practical feedback control systems, which is another source of performance degradation and system instability. For more than two decades, the stability/performance analysis and control design have been extensively studied for linear systems with saturating actuators [6], [7], [21], [30], [32]. If the open-loop system is not exponentially unstable, the semi-global/global stabilization can

This work was supported by the National Natural Science Foundation of China under Grants 61773156, 61873148 and 61933007, the Program for Science and Technology Innovation Talents in the Universities of Henan Province of China under Grant 19HASTIT028, the Research Fund for the Taishan Scholar Project of Shandong Province of China, the Royal Society of the U.K., and the Alexander von Humboldt Foundation of Germany. (Corresponding author: Zidong Wang)

Y. Chen is with the School of Electrical Engineering and Automation, Henan Polytechnic University, Jiaozuo 454000, China and also with the School of Mathematical Sciences, Henan Institute of Science and Technology, Xinxiang 453003, China. E-mail: happycygzmdetom.com

Z. Wang is with the College of Electrical Engineering and Automation, Shandong University of Science and Technology, Qingdao 266590, China, and also with the Department of Computer Science, Brunel University London, Uxbridge UB8 3PH, U.K. E-mail: Zidong.Wang@brunel .ac.uk be achieved via some typical approaches such as pole placement, parameterized Riccati equation and low-and-high gain design methods [7], [30]. In case the open-loop system is exponentially unstable, the local/regional analysis and synthesis can be carried out by using the polytopic models and the generalized sector condition [21], [32].

For control systems subject to both time delays and actuator saturations, some pioneering results have appeared in the literature [1], [2], [5], [8], [9], [16], [17], [19], [20], [23], [28], [31]. For example, the local/regional stabilization problem has been investigated in [1], [5], [28] for continuous-time saturated systems with state delays. For discrete-time state-delayed systems with saturating actuators, some remarkable results have been proposed in [2], [16], [17], [19], [23]. In [9], the solution bounds have been obtained within the firstdelay-interval for input-delayed systems and the regional stabilization problem has been subsequently solved under actuator saturations. Nevertheless, it is worth pointing out that most existing results have been obtained for saturated control systems with a single state delay, input delay or output delay. Moreover, in [9], the time-varying input delay has been assumed to be slowly-varying and the technique used to handle the saturation nonlinearity is a bit conservative. Up to now, to the best of our knowledge, the local stabilization problem has not been sufficiently examined for saturated control systems with both state and input delays, not to mention the case that the distributed state delay and the fast-varying input delay are also involved.

Motivated by the above discussions, this paper is devoted to the investigation of the local stabilization problem for discrete-time systems with both distributed state delay and fast-varying input delay under actuator saturations. The main contributions of the paper are summarized as follows. 1) The traditional constraint on the timevarying input delay is removed for saturated input-delay systems and a novel analysis approach is proposed. 2) By introducing the distributed delay terms and the current state term, a new polytopic model is proposed to characterize the delayed saturation nonlinearity. 3) The local stabilization condition and the optimization problem are established for saturated systems with both state and input delays.

Notation. $P>0(\geq 0)$ denotes that $P$ is a real, symmetric, and positive definite (positive semi-definite) matrix. $\mathbb{R}^{n}$ is the $n$ dimensional Euclidean space. $\lambda_{M}(P)$ is the maximum eigenvalue of the matrix $P .\|\cdot\|_{2}$ and $\|\cdot\|_{\infty}$ are the 2-norm and $\infty$-norm of a vector, respectively. $e_{m, k} \in \mathbb{R}^{1 \times m}$ is a row vector whose $k$-th element is 1 and others are zero. $\otimes$ is the Kronecker product. $K_{(l)}$ is the $l$-th row of the matrix $K . I$ is the identity matrix with compatible dimension.

\section{PROBLEM FORMULATION}

Consider the following discrete-time system with distributed state delay, fast-varying input delay and actuator saturations:

$$
\begin{aligned}
x(k+1) & =A x(k)+A_{d} \sum_{i=1}^{+\infty} \mu_{i} x(k-i)+B \operatorname{sat}\left(u\left(k-\tau_{k}\right)\right), \\
x(k) & =\phi(k), k \in(-\infty, 0]
\end{aligned}
$$

(C) 2020 IEEE. Personal use of this material is permitted. Permission from IEEE must be obtained for all other uses, in any current or future media, including reprinting/republishing this material for advertising or promotional purposes, creating new collective works, for resale or redistribution to servers or lists, or reuse of any copyrighted component of this work in other works. 
where $x(k) \in \mathbb{R}^{n}$ denotes the system state; $u(k) \in \mathbb{R}^{m}$ is the control input with $u(k)=0$ for $k<0 ; \phi(k) \in \mathbb{R}^{n}$ is the initial condition; $A, A_{d}$, and $B$ are known real constant matrices; the summation $\sum_{i=1}^{+\infty} \mu_{i} x(k-i)$ represents the distributed state-delay term; and $\tau_{k}$ denotes the time-varying input delay satisfying $0 \leq \tau_{k} \leq \tau$ ( $\tau$ is a positive integer). Here, $\operatorname{sat}(u)=\left[\begin{array}{llll}\operatorname{sat}\left(u_{1}\right) & \operatorname{sat}\left(u_{2}\right) & \cdots & \operatorname{sat}\left(u_{m}\right)\end{array}\right]^{T}$ is the standard saturation function with unity saturation level, where $\operatorname{sat}\left(u_{l}\right)=\operatorname{sgn}\left(u_{l}\right) \min \left\{\left|u_{l}\right|, 1\right\}, l \in[1, m]$.

The initial condition $\phi(k)$ is assumed to belong to the set

$$
\mathscr{X}_{\rho} \triangleq\left\{\phi(k): \max _{k \leq 0}\|\phi(k)\|_{2} \leq \rho_{1}, \max _{k \leq-1}\|\Delta \phi(k)\|_{2} \leq \rho_{2}\right\}
$$

where $\Delta \phi(k)=\phi(k+1)-\phi(k)$, and $\rho_{1}$ and $\rho_{2}$ are positive scalars. Of course, we can also assume that $\phi(k)$ belongs to the set

$$
\tilde{\mathscr{X}}_{\rho} \triangleq\left\{\phi(k): \max _{k \leq 0}\|\phi(k)\|_{2} \leq \rho\right\} .
$$

Remark 1: For local stabilization of saturated systems, the exact characterization of the region of attraction is very difficult. In this paper, the estimate of region of attraction is characterized by the set $\mathscr{X}_{\rho}$ or $\tilde{\mathscr{X}}_{\rho}$. For the set $\mathscr{X}_{\rho}$, the constrains on the 2-norms of $\phi(k)$ and its variation $\Delta \phi(k)$ are required. However, there is no requirement on $\Delta \phi(k)$ for the set $\tilde{\mathscr{X}}_{\rho}$. If one sets $\rho_{2}=2 \rho_{1} \triangleq \rho$ in $\mathscr{X}_{\rho}$, the set $\tilde{\mathscr{X}}_{\rho}$ is recovered [2], [19]. When $\phi(k)$ is slowly-varying with $\rho_{2}<2 \rho_{1}$, the set $\mathscr{X}_{\rho}$ is less conservative than $\tilde{\mathscr{X}}_{\rho}$ in characterizing admissible initial conditions. Some other effective characterizations of admissible initial conditions have been presented in [16], [19].

In this paper, we employ the state feedback controller

$$
u(k)=K x(k), k \geq 0
$$

where $K \in \mathbb{R}^{m \times n}$ is the controller gain matrix.

For the system (1), we make the following assumptions.

Assumption 1: For given scalars $\mu_{i}>0(i=1,2, \ldots)$, there exists a positive scalar $0<\lambda \leq 1$ such that

$$
\sum_{i=1}^{+\infty} \mu_{i} \lambda^{-i}<\sum_{j=1}^{+\infty} \sum_{i=1}^{j} \mu_{j} \lambda^{-i}<\sum_{l=1}^{+\infty} \sum_{j=1}^{l} \sum_{i=1}^{j} \mu_{l} \lambda^{-i}<+\infty .
$$

Assumption 2: There exists a time sequence

$$
0=k_{1}<\bar{k}_{1}<k_{2}<\bar{k}_{2}<\cdots<k_{r}<\bar{k}_{r}=k^{*} \leq \tau
$$

such that the following relationship holds:

$$
k-\tau_{k}<0, k \in \mathcal{T}_{\uparrow} ; k-\tau_{k} \geq 0, k \in \mathcal{T}_{\downarrow} \cup\left[k^{*},+\infty\right)
$$

where

$$
\begin{aligned}
& \mathcal{T}_{\uparrow} \triangleq\left[k_{1}, \bar{k}_{1}\right) \cup\left[k_{2}, \bar{k}_{2}\right) \cup \cdots \cup\left[k_{r}, \bar{k}_{r}\right), \\
& \mathcal{T}_{\downarrow} \triangleq\left[\bar{k}_{1}, k_{2}\right) \cup\left[\bar{k}_{2}, k_{3}\right) \cup \cdots \cup\left[\bar{k}_{r-1}, k_{r}\right) .
\end{aligned}
$$

Remark 2: In Assumption 2, the time sequence does not have to be exactly known. Here, we only require that $k^{*}$ is known (or unknown but upper-bounded by a known integer $\tau^{*} \leq \tau$ ). In [9], it is assumed that there exists a unique integer $k^{*}$ such that $k-\tau_{k}<0$ for $k \in$ $\left[0, k^{*}\right)$ and $k-\tau_{k} \geq 0$ for $k \in\left[k^{*},+\infty\right)$. Such an assumption requires the input delay $\tau_{k}$ to be slowly-varying with $\left|\tau_{k+1}-\tau_{k}\right| \leq 1$. From Assumption 2, it is seen that $\tau_{k}$ can be fast-varying in this paper since the constraint $\left|\tau_{k+1}-\tau_{k}\right| \leq 1$ is no longer necessary.

Under Assumption 2, there will be no control signal (i.e, $u(k-$ $\left.\tau_{k}\right)=0$ ) within the interval $\mathcal{T}_{\uparrow}$ due to the time delay of the control input. In this case, the system (1) is equivalent to

$$
x(k+1)=A x(k)+A_{d} \sum_{i=1}^{+\infty} \mu_{i} x(k-i), k \in \mathcal{T}_{\uparrow} .
$$

To handle the actuator saturations within the interval $\mathcal{T}_{\downarrow}$, as in [6], we define the dead-zone nonlinearity $\psi(u(k)) \triangleq u(k)-\operatorname{sat}(u(k))$. Adding and subtracting $u\left(k-\tau_{k}\right)$ in the right side of (1) yields

$$
\begin{aligned}
x(k+1)= & A x(k)+B K x\left(k-\tau_{k}\right)+A_{d} \sum_{i=1}^{+\infty} \mu_{i} x(k-i) \\
& -B \psi\left(u\left(k-\tau_{k}\right)\right), k \in \mathcal{T}_{\downarrow} .
\end{aligned}
$$

Moreover, the following (classical) sector condition holds [6], [21]:

$$
\psi^{T}\left(u\left(k-\tau_{k}\right)\right) H\left[\psi\left(u\left(k-\tau_{k}\right)\right)-K x\left(k-\tau_{k}\right)\right] \leq 0, k \in \mathcal{T}_{\downarrow}
$$

where $H \in \mathbb{R}^{m \times m}$ is any positive diagonal matrix.

Next, we will introduce two important lemmas.

Lemma 1: [32] Let $v \in \mathbb{R}^{\overleftrightarrow{m}}$ be such that $\|v\|_{\infty} \leq 1$ where $\stackrel{\leftrightarrow}{m}=$ $m 2^{m-1}$. Let the elements in $\mathbb{D}_{m}$ be labeled as $D_{i}\left(i \in\left[1,2^{m}\right]\right)$ where $\mathbb{D}_{m}$ is a set of $m \times m$ diagonal matrices with diagonal elements being either 1 or 0 , and the function $f_{m}$ be defined as $f_{m}(0)=0$ and

$$
f_{m}(i)=\left\{\begin{array}{ll}
f_{m}(i-1)+1, & D_{i}+D_{j} \neq I_{m}, \quad \forall j \in[1, i] \\
f_{m}(j), & D_{i}+D_{j}=I_{m}, \quad \exists j \in[1, i]
\end{array} .\right.
$$

Then, for any $u \in \mathbb{R}^{m}$, there holds $\operatorname{sat}(u) \in \operatorname{co}\left\{D_{i} u+\mathcal{D}_{i}^{-} v: i \in\right.$ $\left.\left[1,2^{m}\right]\right\}$, where "co" denotes the convex hull and $\mathcal{D}_{i}^{-} \in \mathbb{R}^{m \times \stackrel{\leftrightarrow}{m}}$ is defined as $\mathcal{D}_{i}^{-}=e_{2^{m-1}, f_{m}(i)} \otimes D_{i}^{-}$with $D_{i}^{-}=I-D_{i}$.

Lemma 2: [2], [18] Let $0<Z \in \mathbb{R}^{n \times n}, x_{i} \in \mathbb{R}^{n}$ and the scalars $\mu_{i} \geq 0, \lambda_{j} \geq 0(i, j=1,2, \cdots)$ be given. Then, we have

$$
\begin{aligned}
\text { 1) } & \left(\sum_{i=1}^{+\infty} \mu_{i} x_{i}\right)^{T} Z\left(\sum_{i=1}^{+\infty} \mu_{i} x_{i}\right) \\
\leq & \left(\sum_{i=1}^{+\infty} \mu_{i} \lambda_{i}^{-1}\right)\left(\sum_{i=1}^{+\infty} \mu_{i} \lambda_{i} x_{i}^{T} Z x_{i}\right), \\
\text { 2) } & \left(\sum_{j=1}^{+\infty} \sum_{i=1}^{j} \mu_{j} x_{i}\right)^{T} Z\left(\sum_{j=1}^{+\infty} \sum_{i=1}^{j} \mu_{j} x_{i}\right) \\
\leq & \left(\sum_{j=1}^{+\infty} \sum_{i=1}^{j} \mu_{j} \lambda_{i}^{-1}\right)\left(\sum_{j=1}^{+\infty} \sum_{i=1}^{j} \mu_{j} \lambda_{i} x_{i}^{T} Z x_{i}\right) .
\end{aligned}
$$

Now, let $U, V$ and $W$ be $\stackrel{\leftrightarrow}{m} \times n$ matrices and denote that

$$
v(k) \triangleq U x(k)+V \sum_{i=1}^{+\infty} \mu_{i} x(k-i)+W \sum_{j=1}^{+\infty} \mu_{j} \sum_{i=k-j}^{k-1} x(i) .
$$

Assume that the following constraint condition holds:

$$
\|v(k)\|_{\infty} \leq 1, k \in\left[k^{*},+\infty\right) .
$$

Then, from Lemma 1 , sat $\left(u\left(k-\tau_{k}\right)\right)$ can be represented as

$$
\operatorname{sat}\left(u\left(k-\tau_{k}\right)\right)=\sum_{s=1}^{2^{m}} \varpi_{s}^{k}\left[D_{s} u\left(k-\tau_{k}\right)+\mathcal{D}_{s}^{-} v(k)\right]
$$

where $k \in\left[k^{*},+\infty\right), \varpi_{s}^{k} \geq 0\left(s \in\left[1,2^{m}\right]\right)$ and $\sum_{s=1}^{2^{m}} \varpi_{s}^{k}=1$.

We are now ready to present the closed-loop system as follows:

$$
\begin{aligned}
x(k+1)= & \sum_{s=1}^{2^{m}} \varpi_{s}^{k}\left\{\left(A+B \mathcal{D}_{s}^{-} U\right) x(k)+B D_{s} K\right. \\
& \times x\left(k-\tau_{k}\right)+\left(A_{d}+B \mathcal{D}_{s}^{-} V\right) \sum_{i=1}^{+\infty} \mu_{i} x(k-i) \\
& \left.+B \mathcal{D}_{s}^{-} W \sum_{j=1}^{+\infty} \mu_{j} \sum_{i=k-j}^{k-1} x(i)\right\}, k \in\left[k^{*},+\infty\right) .
\end{aligned}
$$

Remark 3: To deal with the delayed saturation nonlinearity in a less conservative framework, the distributed-delay-dependent terms 
$\mathcal{D}_{s}^{-} V \sum_{i=1}^{h} \mu_{i} x(k-i)$ and $\mathcal{D}_{s}^{-} W \sum_{j=1}^{h} \mu_{j} \sum_{i=k-j}^{k-1} x(i)$ are additionally introduced in the polytopic model (11). Furthermore, it is worth mentioning that, different from the sector condition used in [4] (pp. 239), our proposed polytopic model (11) utilizes the current state $x(k)$ of the system (1), thereby facilitating the reduction of the possible conservatism. In addition, it is worth mentioning that the closed-loop system has different representations within the intervals $\mathcal{T}_{\downarrow}$ and $\left[k^{*},+\infty\right)$. Using the model (7), the global analysis can be performed within $\mathcal{T}_{\downarrow}$ and using (12), the local analysis can be done in a less conservative manner for the multiple-input case [32].

This paper aims to design the controller (5) such that the closedloop system (12) is locally exponentially stable with an estimate of the region of attraction that is made as large as possible.

\section{MAin Results}

For the purpose of exponential stability analysis, we first propose the following piecewise augmented Lyapunov functional:

$$
V(k)= \begin{cases}V_{1}(k), & k \in\left[k^{*},+\infty\right), \\ V_{2}(k), & k \in\left[0, k^{*}\right)\end{cases}
$$

where

$$
\begin{aligned}
V_{\alpha}(k)= & \eta^{T}(k) P_{\alpha} \eta(k)+\sum_{i=k-\tau}^{k-1} \lambda_{\alpha}^{k-i-1} x^{T}(i) Q_{\alpha} x(i) \\
& +\sum_{j=1}^{+\infty} \mu_{j} \sum_{i=k-j}^{k-1} \lambda_{\alpha}^{k-i-1} x^{T}(i) S_{\alpha 1} x(i) \\
& +\sum_{l=1}^{+\infty} \mu_{l} \sum_{j=1}^{l} \sum_{i=k-j}^{k-1} \lambda_{\alpha}^{k-i-1} x^{T}(i) S_{\alpha 2} x(i) \\
& +\tau \sum_{j=-\tau}^{-1} \sum_{i=k+j}^{k-1} \lambda_{\alpha}^{k-i-1} y^{T}(i)\left(R_{\alpha 1}+R_{\alpha 2}\right) y(i) \\
& +\sum_{l=1}^{+\infty} \mu_{l} \sum_{j=1}^{l} \sum_{i=k-j}^{k-1} \lambda_{\alpha}^{k-i-1} y^{T}(i) Z_{\alpha} y(i), \alpha=1,2
\end{aligned}
$$

with $P_{\alpha}>0, Q_{\alpha}>0, S_{\alpha 1}>0, S_{\alpha 2}>0, R_{\alpha 1}>0, R_{\alpha 2}>0$, $Z_{\alpha}>0,0<\lambda_{1} \leq 1, \lambda_{2}>1, y(k)=x(k+1)-x(k)$, and $\eta(k)=\left[\begin{array}{lll}x^{T}(k) & \sum_{i=k-\tau}^{\bar{k}-1} x^{T}(i) & \sum_{j=1}^{+\infty} \mu_{j} \sum_{i=k-j}^{k-1} x^{T}(i)\end{array}\right]^{T}$.

For convenience of subsequent presentation, we define

$$
\begin{aligned}
& \check{\tau}_{k} \triangleq \tau_{k}+1, \hat{\tau}_{k} \triangleq \tau-\tau_{k}+1, \tilde{\tau} \triangleq \tau+1, \\
& \xi_{0}(k) \triangleq\left[\sum_{i=1}^{+\infty} \mu_{i} x^{T}(k-i) \sum_{j=1}^{+\infty} \mu_{j} \sum_{i=k-j}^{k-1} x^{T}(i) y^{T}(k)\right]^{T}, \\
& \xi_{1}(k) \triangleq\left[x^{T}(k) \quad x^{T}\left(k-\tau_{k}\right) \quad x^{T}(k-\tau)\right. \\
& \left.\frac{1}{\check{\tau}_{k}} \sum_{i=k-\tau_{k}}^{k} x^{T}(i) \frac{1}{\hat{\tau}_{k}} \sum_{i=k-\tau}^{k-\tau_{k}} x^{T}(i) \xi_{0}^{T}(k)\right]^{T}, \\
& \xi_{2}(k) \triangleq\left[\begin{array}{lll}
x^{T}(k) & x^{T}\left(k-\tau_{k}\right) & x^{T}(k-\tau)
\end{array}\right. \\
& \left.\frac{1}{\tilde{\tau}} \sum_{i=k-\tau}^{k} x^{T}(i) \quad \xi_{0}^{T}(k) \psi^{T}\left(u\left(k-\tau_{k}\right)\right)\right]^{T}, \\
& \xi_{3}(k) \triangleq\left[\begin{array}{llll}
x^{T}(k) & x^{T}(k-\tau) & \frac{1}{\tilde{\tau}} \sum_{i=k-\tau}^{k} x^{T}(i) & \xi_{0}^{T}(k)
\end{array}\right]^{T}, \\
& \Gamma_{1} \triangleq\left[\begin{array}{cccccccc}
I & 0 & 0 & 0 & 0 & 0 & 0 & I \\
0 & -I & -I & \check{\tau}_{k} I & \hat{\tau}_{k} I & 0 & 0 & 0 \\
\kappa I & 0 & 0 & 0 & 0 & -I & I & 0
\end{array}\right] \text {, } \\
& \Gamma_{2} \triangleq\left[\begin{array}{cccccccc}
I & 0 & 0 & 0 & 0 & 0 & 0 & 0 \\
-I & -I & 0 & \check{\tau}_{k} I & \hat{\tau}_{k} I & 0 & 0 & 0 \\
0 & 0 & 0 & 0 & 0 & 0 & I & 0
\end{array}\right]
\end{aligned}
$$

$$
\begin{aligned}
& \Gamma_{3} \triangleq\left[\begin{array}{cccccccc}
I & 0 & 0 & 0 & 0 & 0 & I & 0 \\
0 & 0 & -I & \tilde{\tau} I & 0 & 0 & 0 & 0 \\
\kappa I & 0 & 0 & 0 & -I & I & 0 & 0
\end{array}\right], \\
& \Gamma_{4} \triangleq\left[\begin{array}{ccccccc}
I & 0 & 0 & 0 & 0 & 0 & 0_{n \times(n+m)} \\
-I & 0 & 0 & \tilde{\tau} I & 0 & 0 & 0_{n \times(n+m)} \\
0 & 0 & 0 & 0 & 0 & I & 0_{n \times(n+m)}
\end{array}\right] \text {, } \\
& \Gamma_{5} \triangleq\left[\begin{array}{cccccc}
I & 0 & 0 & 0 & 0 & I \\
0 & -I & \tilde{\tau} I & 0 & 0 & 0 \\
\kappa I & 0 & 0 & -I & I & 0
\end{array}\right] \\
& \Gamma_{6} \triangleq\left[\begin{array}{cccccc}
I & 0 & 0 & 0 & 0 & 0 \\
-I & 0 & \tilde{\tau} I & 0 & 0 & 0 \\
0 & 0 & 0 & 0 & I & 0
\end{array}\right] \\
& \Phi_{1} \triangleq\left[\begin{array}{cccccc}
I & -I & 0 & 0 & 0 & 0_{n \times 3 n} \\
I & I & 0 & -2 I & 0 & 0_{n \times 3 n} \\
0 & I & -I & 0 & 0 & 0_{n \times 3 n} \\
0 & I & I & 0 & -2 I & 0_{n \times 3 n}
\end{array}\right], \\
& \Phi_{2} \triangleq\left[\begin{array}{llll}
\kappa I & 0_{n \times 4 n} & -I & 0_{n \times 2 n}
\end{array}\right], \\
& \Phi_{3} \triangleq\left[\begin{array}{cccc}
I & -I & 0 & 0_{n \times(4 n+m)} \\
0 & I & -I & 0_{n \times(4 n+m)}
\end{array}\right], \\
& \Phi_{4} \triangleq\left[\begin{array}{ccccc}
I & 0 & -I & 0 & 0_{n \times(3 n+m)} \\
I & 0 & I & -2 I & 0_{n \times(3 n+m)}
\end{array}\right], \\
& \Phi_{5} \triangleq\left[\begin{array}{llll}
\kappa I & 0_{n \times 3 n} & -I & 0_{n \times(2 n+m)}
\end{array}\right], \\
& \Phi_{6} \triangleq\left[\begin{array}{cccc}
I & -I & 0 & 0_{n \times 3 n} \\
I & I & -2 I & 0_{n \times 3 n}
\end{array}\right], R_{\alpha} \triangleq R_{\alpha 1}+R_{\alpha 2}, \\
& \Phi_{7} \triangleq\left[\begin{array}{llll}
\kappa I & 0_{n \times 2 n} & -I & 0_{n \times 2 n}
\end{array}\right], \bar{R}_{\alpha} \triangleq \bar{R}_{\alpha 1}+\bar{R}_{\alpha 2}, \\
& \Phi_{8} \triangleq\left[\begin{array}{lll}
\tau I & -I & 0_{n \times 2 n}
\end{array}\right], \Phi_{9} \triangleq\left[\begin{array}{lll}
\kappa I & 0_{n \times 2 n} & -I
\end{array}\right], \\
& \kappa \triangleq \sum_{i=1}^{+\infty} \mu_{i}, \tilde{\kappa}_{\alpha} \triangleq \sum_{i=1}^{+\infty} \mu_{i} \lambda_{\alpha}^{-i}, \sigma \triangleq \sum_{i=1}^{+\infty} i \mu_{i}, \\
& \tilde{\sigma}_{\alpha} \triangleq \sum_{j=1}^{+\infty} \sum_{i=1}^{j} \mu_{j} \lambda_{\alpha}^{-i}, \operatorname{Sym}(\mathcal{E}) \triangleq \mathcal{E}+\mathcal{E}^{T}, \\
& \varphi_{1} \triangleq \frac{\lambda_{2}^{\tau}-1}{\lambda_{2}-1}, \varphi_{4} \triangleq \frac{\left(1-\lambda_{2}\right) \tau-\lambda_{2}+\lambda_{2}^{\tau+1}}{\left(\lambda_{2}-1\right)^{2}}, \\
& \varphi_{2 \alpha} \triangleq \sum_{j=1}^{+\infty} \sum_{i=1}^{j} \mu_{j} \lambda_{\alpha}^{i-1}, \varphi_{3 \alpha} \triangleq \sum_{l=1}^{+\infty} \sum_{j=1}^{l} \sum_{i=1}^{j} \mu_{l} \lambda_{\alpha}^{i-1} .
\end{aligned}
$$

Theorem 1: Let the scalars $0<\lambda_{1} \leq 1, \lambda_{2}>1, \nu>0$, the integer $k^{*} \geq 1$ and the matrix $K$ be given. Assume that there exist matrices $0<P_{\alpha} \in \mathbb{R}^{3 n \times 3 n}, 0<Q_{\alpha} \in \mathbb{R}^{n \times n}, 0<S_{\alpha j} \in \mathbb{R}^{n \times n}$, $0<R_{\alpha j} \in \mathbb{R}^{n \times n}, 0<Z_{\alpha} \in \mathbb{R}^{n \times n}, T_{i j} \in \mathbb{R}^{n \times n}(\alpha, j=1,2$, $i=1,2,3), M_{1} \in \mathbb{R}^{2 n \times 2 n}, M_{2} \in \mathbb{R}^{n \times n}, U \in \mathbb{R}^{\overleftrightarrow{m} \times n}, V \in \mathbb{R}^{\overleftrightarrow{m} \times n}$, $W \in \mathbb{R}^{\overleftrightarrow{m} \times n}$, and the diagonal matrix $0<H \in \mathbb{R}^{m \times m}$ such that, for $\tau_{k}=0, \tau, \forall s \in\left[1,2^{m}\right], \forall l \in[1, \overleftrightarrow{m}]$, the matrix inequalities

$$
\begin{aligned}
& \Lambda_{1} \triangleq\left[\begin{array}{cc}
\check{R}_{1} & M_{1} \\
M_{1}^{T} & \check{R}_{1}
\end{array}\right]>0, \Lambda_{2} \triangleq\left[\begin{array}{cc}
R_{21} & M_{2} \\
M_{2}^{T} & R_{21}
\end{array}\right]>0, \\
& \Xi_{1}\left(\tau_{k}, s\right) \triangleq \Gamma_{1}^{T} P_{1} \Gamma_{1}-\lambda_{1} \Gamma_{2}^{T} P_{1} \Gamma_{2}-\lambda_{1}^{\tau} \Phi_{1}^{T} \Lambda_{1} \Phi_{1} \\
& \quad-\Phi_{2}^{T}\left(Z_{1} / \tilde{\sigma}_{1}\right) \Phi_{2}+\operatorname{Sym}\left(T_{1} \Sigma_{1}\right)+\Psi_{1}<0, \\
& \Xi_{2} \triangleq \Gamma_{3}^{T} P_{2} \Gamma_{3}-\lambda_{2}\left(\Gamma_{4}^{T} P_{2} \Gamma_{4}+\Phi_{3}^{T} \Lambda_{2} \Phi_{3}+\Phi_{4}^{T} \check{R}_{22}^{\tau} \Phi_{4}\right) \\
& \quad-\Phi_{5}^{T}\left(Z_{2} / \tilde{\sigma}_{2}\right) \Phi_{5}+\operatorname{Sym}\left(T_{2} \Sigma_{2}+T_{4} \Sigma_{4}\right)+\Psi_{2}<0, \\
& \Xi_{3} \triangleq \Gamma_{5}^{T} P_{2} \Gamma_{5}-\lambda_{2} \Gamma_{6}^{T} P_{2} \Gamma_{6}-\lambda_{2} \Phi_{6}^{T} \check{R}_{2}^{\tau} \Phi_{6} \\
& -\Phi_{7}^{T}\left(Z_{2} / \tilde{\sigma}_{2}\right) \Phi_{7}+\operatorname{Sym}\left(T_{3} \Sigma_{3}\right)+\Psi_{3}<0, \\
& \left\{\begin{array}{c}
P_{1} \leq \nu P_{2}, Q_{1} \leq \nu Q_{2}, S_{1 j} \leq \nu S_{2 j}, \\
R_{1 j} \leq \nu R_{2 j}(j=1,2), Z_{1} \leq \nu Z_{2},
\end{array}\right. \\
& \Xi_{4}(l) \triangleq\left[\begin{array}{cc}
1 /\left(\nu \lambda_{2}^{k^{*}}\right) & N_{(l)} \\
N_{(l)}^{T} & \operatorname{diag}\left\{P_{1}, 0\right\}+\Psi_{4} / \lambda_{1}
\end{array}\right] \geq 0,
\end{aligned}
$$


This article has been accepted for publication in a future issue of this journal, but has not been fully edited. Content may change prior to final publication. Citation information: DOI10.1109/TAC.2020.2991013, IEEE Transactions on Automatic Control

are satisfied, where $N_{(l)}=\left[\begin{array}{lllll}U_{(l)} & 0 & W_{(l)} & V_{(l)}\end{array}\right]$ and

$$
\begin{aligned}
& \Psi_{1}=\operatorname{diag}\left\{Q_{1}+\kappa S_{11}+\sigma S_{12}, 0,-\lambda_{1}^{\tau} Q_{1}, 0,\right. \\
& \left.0,-S_{11} / \tilde{\kappa}_{1},-S_{12} / \tilde{\sigma}_{1}, \tau^{2} R_{1}+\sigma Z_{1}\right\}, \\
& \Psi_{2}=\operatorname{diag}\left\{Q_{2}+\kappa S_{21}+\sigma S_{22}, 0,-\lambda_{2}^{\tau} Q_{2}, 0,\right. \\
& \left.-S_{21} / \tilde{\kappa}_{2},-S_{22} / \tilde{\sigma}_{2}, \tau^{2} R_{2}+\sigma Z_{2}, 0\right\}, \\
& \Psi_{3}=\operatorname{diag}\left\{Q_{2}+\kappa S_{21}+\sigma S_{22},-\lambda_{2}^{\tau} Q_{2}, 0,\right. \\
& \left.-S_{21} / \tilde{\kappa}_{2},-S_{22} / \tilde{\sigma}_{2}, \tau^{2} R_{2}+\sigma Z_{2}\right\}, \\
& \Psi_{4}=\operatorname{diag}\left\{0,\left(\lambda_{1}^{\tau} / \tau\right) Q_{1}, S_{12} / \tilde{\sigma}_{1}, S_{11} / \tilde{\kappa}_{1}\right\} \\
& +\left(2 \lambda_{1}^{\tau} / \tilde{\tau}\right) \Phi_{8}^{T} R_{1} \Phi_{8}+\left(1 / \tilde{\sigma}_{1}\right) \Phi_{9} Z_{1} \Phi_{9}, \\
& T_{1}=\left[\begin{array}{lll}
T_{11}^{T} & 0_{n \times 6 n} & T_{12}^{T}
\end{array}\right]^{T}, T_{3}=\left[\begin{array}{lll}
T_{31}^{T} & 0_{n \times 4 n} & T_{32}^{T}
\end{array}\right]^{T}, \\
& T_{2}=\left[\begin{array}{llll}
T_{21}^{T} & 0_{n \times 5 n} & T_{22}^{T} & 0_{n \times m}
\end{array}\right]^{T}, T_{4}=\left[\begin{array}{ll}
0_{m \times 7 n} & H^{T}
\end{array}\right]^{T}, \\
& \Sigma_{1}=\left[\begin{array}{llll}
A+B \mathcal{D}_{s}^{-} U-I & B D_{s} K & 0_{n \times 3 n}
\end{array}\right. \\
& \left.A_{d}+B \mathcal{D}_{s}^{-} V \quad B \mathcal{D}_{s}^{-} W \quad-I\right], \\
& \Sigma_{2}=\left[\begin{array}{lllllll}
A-I & B K & 0_{n \times 2 n} & A_{d} & 0_{n \times n} & -I & -B
\end{array}\right], \\
& \Sigma_{3}=\left[\begin{array}{lllll}
A-I & 0_{n \times 2 n} & A_{d} & 0_{n \times n} & -I
\end{array}\right], \\
& \Sigma_{4}=\left[\begin{array}{llll}
0_{m \times n} & K & 0_{m \times 5 n} & -I
\end{array}\right], \check{R}_{1} \triangleq \operatorname{diag}\left\{R_{1}, 3 R_{1}\right\}, \\
& \check{R}_{2}^{\tau} \triangleq \operatorname{diag}\left\{R_{2}, 3 \varphi_{\tau} R_{2}\right\}, \check{R}_{22}^{\tau} \triangleq \operatorname{diag}\left\{R_{22}, 3 \varphi_{\tau} R_{22}\right\}, \\
& \left(\varphi_{\tau} \triangleq(\tau+1) /(\tau-1)(\tau>1), \varphi_{1} \triangleq 1\right) \text {. }
\end{aligned}
$$

Then, for any initial condition $\phi(k) \in \mathscr{X}_{\rho}$ satisfying $V_{2}(0) \leq 1$, the closed-loop system (12) is exponentially stable.

Proof: Letting $\triangle V_{\alpha}(k) \triangleq V_{\alpha}(k+1)-\lambda_{\alpha} V_{\alpha}(k)(\alpha=1,2)$, by calculations and using Lemma 2 , it follows that

$$
\begin{aligned}
\triangle V_{\alpha}(k) & \leq \eta^{T}(k+1) P_{\alpha} \eta(k+1)-\lambda_{\alpha} \eta^{T}(k) P_{\alpha} \eta(k) \\
& +x^{T}(k)\left(Q_{\alpha}+\kappa S_{\alpha 1}+\sigma S_{\alpha 2}\right) x(k)+y^{T}(k) \\
& \times\left(\tau^{2} R_{\alpha}+\sigma Z_{\alpha}\right) y(k)-\lambda_{\alpha}^{\tau} x^{T}(k-\tau) Q_{\alpha} x(k-\tau) \\
& -\zeta_{1}^{T}(k)\left(S_{\alpha 1} / \tilde{\kappa}_{\alpha}\right) \zeta_{1}(k)-\zeta_{2}^{T}(k)\left(S_{\alpha 2} / \tilde{\sigma}_{\alpha}\right) \zeta_{2}(k) \\
& -\tau \tilde{\lambda}_{\alpha} \sum_{i=k-\tau}^{k-1} y^{T}(i) R_{\alpha} y(i)-\zeta_{3}^{T}(k)\left(Z_{\alpha} / \tilde{\sigma}_{\alpha}\right) \zeta_{3}^{T}(k)
\end{aligned}
$$

where $\tilde{\lambda}_{1}=\lambda_{1}^{\tau}, \tilde{\lambda}_{2}=\lambda_{2}, \zeta_{1}(k)=\sum_{i=1}^{+\infty} \mu_{i} x(k-i), \zeta_{2}(k)=$ $\sum_{j=1}^{+\infty} \sum_{i=1}^{j} \mu_{j} x(k-i)$, and $\zeta_{3}(k)=\sum_{j=1}^{+\infty} \sum_{i=1}^{j} \mu_{j} y(k-i)$.

Using the discrete Wirtinger-based inequality [2], [14] and the reciprocally convex combination inequality [14], and noting the fact $\sum_{i=k-\tau}^{k-1}(\cdot)=\sum_{i=k-\tau_{k}}^{k-1}(\cdot)+\sum_{i=k-\tau}^{k-\tau_{k}-1}(\cdot)$, we have

$$
\tau \sum_{i=k-\tau}^{k-1} y^{T}(i) R_{1} y(i) \geq \xi_{1}^{T}(k) \Phi_{1}^{T} \Lambda_{1} \Phi_{1} \xi_{1}(k)
$$

subject to the constraint $\Lambda_{1}>0$ (the first inequality in (14)). Similarly, using the Jensen inequality [2] and the reciprocally convex combination inequality [14], it follows that

$$
\tau \sum_{i=k-\tau}^{k-1} y^{T}(i) R_{21} y(i) \geq \xi_{2}^{T}(k) \Phi_{3}^{T} \Lambda_{2} \Phi_{3} \xi_{2}(k)
$$

where the matrix $\Lambda_{2}$ given in (14) satisfies $\Lambda_{2}>0$. In particular, using Wirtinger-based inequality directly, one obtains

$$
\begin{gathered}
\tau \sum_{i=k-\tau}^{k-1} y^{T}(i) R_{22} y(i) \geq \xi_{2}^{T}(k) \Phi_{4}^{T} \check{R}_{22}^{\tau} \Phi_{4} \xi_{2}(k), \\
\tau \sum_{i=k-\tau}^{k-1} y^{T}(i) R_{2} y(i) \geq \xi_{3}^{T}(k) \Phi_{6}^{T} \check{R}_{2}^{\tau} \Phi_{6} \xi_{3}(k) .
\end{gathered}
$$

Noting the matrices $T_{i}$ and $\Sigma_{i}(i=1,2,3)$ that are denoted in the statement of the theorem, we obtain from (12), (7) and (6) that

$$
2 \xi_{1}^{T}(k) T_{1} \Sigma_{1} \xi_{1}(k)=0, k \in\left[k^{*},+\infty\right),
$$

$$
\begin{aligned}
& 2 \xi_{2}^{T}(k) T_{2} \Sigma_{2} \xi_{2}(k)=0, \quad k \in \mathcal{T}_{\downarrow}, \\
& 2 \xi_{3}^{T}(k) T_{3} \Sigma_{3} \xi_{3}(k)=0, \quad k \in \mathcal{T}_{\uparrow} .
\end{aligned}
$$

In addition, it is seen from the sector condition (8) that

$$
-2 \xi_{2}^{T}(k) T_{4} \Sigma_{4} \xi_{2}(k) \geq 0, k \in \mathcal{T}_{\downarrow} .
$$

Adding the left-hand side of (25) to $\Delta V_{1}(k)$, and using (21) and $\sum_{j=1}^{+\infty} \sum_{i=1}^{j} \mu_{j} y(k-i)=\kappa x(k)-\sum_{i=1}^{+\infty} \mu_{j} x(k-i)$, we obtain

$$
\Delta V_{1}(k) \leq \sum_{s=1}^{2^{m}} \varpi_{s}^{k} \xi_{1}^{T}(k) \Xi_{1}\left(\tau_{k}, s\right) \xi_{1}(k), k \in\left[k^{*},+\infty\right)
$$

where $\Xi_{1}\left(\tau_{k}, s\right)$ is defined in (15). Similarly, adding the left-hand sides of (26) and (28) to $\Delta V_{2}(k)$ and using (22)-(23), we have

$$
\Delta V_{2}(k) \leq \xi_{2}^{T}(k) \Xi_{2} \xi_{2}(k), k \in \mathcal{T}_{\downarrow}
$$

where $\Xi_{2}$ is given in the matrix inequality (16). Also, adding the left side of (27) to $\Delta V_{2}(k)$ and applying the inequality (24) yields

$$
\Delta V_{2}(k) \leq \xi_{3}^{T}(k) \Xi_{3} \xi_{3}(k), k \in \mathcal{T}_{\uparrow}
$$

where the matrix $\Xi_{3}$ is denoted in the inequality (17).

For $\tau_{k}=0, \tau$ and $\forall s \in\left[1,2^{m}\right]$, if the matrix inequalities (14)-(17) are satisfied, then we can obtain from (29)-(31) that

$$
\begin{aligned}
& V_{1}(k+1) \leq \lambda_{1} V_{1}(k), \quad k \in\left[k^{*},+\infty\right), \\
& V_{2}(k+1) \leq \lambda_{2} V_{2}(k), \quad k \in\left[0, k^{*}\right) .
\end{aligned}
$$

Moreover, it is seen from the matrix inequalities in (18) that

$$
V_{1}(k) \leq \nu V_{2}(k), k \geq 0
$$

Using the inequalities (32)-(34), it follows that

$$
V_{1}(k) \leq \lambda_{1}^{k-k^{*}}\left[\nu \lambda_{2}^{k^{*}} V_{2}(0)\right], k \in\left[k^{*},+\infty\right) .
$$

On the other hand, noting the following facts:

$$
\begin{aligned}
& \sum_{j=1}^{+\infty} \mu_{j} \sum_{i=k-j}^{k-1} f(i) \geq \sum_{j=1}^{+\infty} \mu_{j} f(k-j), \\
& \sum_{l=1}^{+\infty} \mu_{l} \sum_{j=1}^{l} \sum_{i=k-j}^{k-1} f(i) \geq \sum_{l=1}^{+\infty} \mu_{l} \sum_{j=1}^{l} f(k-j)
\end{aligned}
$$

where $f(i)$ is a positive real function, and using Lemma 2 and the Jensen inequality [2], one has the following inequality:

$$
V_{1}(k) \geq \tilde{\eta}^{T}(k)\left[\operatorname{diag}\left\{P_{1}, 0\right\}+\Psi_{4} / \lambda_{1}\right] \tilde{\eta}(k), k \in\left[k^{*},+\infty\right)
$$

where $\Psi_{4}$ is given in (19) and $\tilde{\eta}(k) \triangleq\left[\begin{array}{lll}\eta^{T}(k) & \sum_{i=1}^{+\infty} \mu_{i} x^{T}(k-i)\end{array}\right]^{T}$. Applying Schur complement to (19), it is clear that

$$
\operatorname{diag}\left\{P_{1}, 0\right\}+\Psi_{4} / \lambda_{1} \geq \nu \lambda_{2}^{k^{*}} N_{(l)}^{T} N_{(l)}, l \in[1, \stackrel{\leftrightarrow}{m}] .
$$

Then, one obtains from (35)-(37) that

$$
\begin{aligned}
\left|v_{l}(k)\right|^{2} & =\tilde{\eta}^{T}(k) N_{(l)}^{T} N_{(l)} \tilde{\eta}(k) \leq\left(1 /\left(\nu \lambda_{2}^{k^{*}}\right)\right) V_{1}(k), \\
& \leq V_{2}(0), \quad l \in[1, \stackrel{\leftrightarrow}{m}], k \in\left[k^{*},+\infty\right) .
\end{aligned}
$$

For any initial condition $\phi(k) \in \mathscr{X}_{\rho}$ satisfying $V_{2}(0) \leq 1$, it is clear from (38) that the constraint condition (10) is ensured. Moreover, one can conclude from (35) that the closed-loop system (12) is locally exponentially stable and this completes the proof.

Remark 4: In the proof of Theorem 1, the global analysis is performed within the interval $\mathcal{T}_{\downarrow}$ by using the classical sector condition (8) since $\mathcal{T}_{\downarrow}$ is generally small and not exactly known. Considering that the open-loop of the system (1) might be unstable, the functional $V_{2}(k)$ along the closed-loop system (7) is required to be increasing. 
Of course, if the sequence $k_{1}, \bar{k}_{1}, k_{2}, \bar{k}_{2}, \cdots, k_{r}, \bar{k}_{r}$ in Assumption 2 is known, one can perform the less conservative local analysis.

Next, we will discuss the controller design in terms of LMIs.

Theorem 2: Let the scalars $0<\lambda_{1} \leq 1, \lambda_{2}>1, \nu>0, \delta_{i} \neq 0$ $(i=1,2,3)$ and the integer $k^{*} \geq 1$ be given. Assume that there exist matrices $0<\bar{P}_{\alpha} \in \mathbb{R}^{3 n \times 3 n}, 0<\bar{Q}_{\alpha} \in \mathbb{R}^{n \times n}, 0<\bar{S}_{\alpha j} \in \mathbb{R}^{n \times n}$, $0<\bar{R}_{\alpha j} \in \mathbb{R}^{n \times n}, 0<\bar{Z}_{\alpha} \in \mathbb{R}^{n \times n}(\alpha, j=1,2), \bar{M}_{1} \in \mathbb{R}^{2 n \times 2 n}$, $\bar{M}_{2} \in \mathbb{R}^{n \times n}, X \in \mathbb{R}^{n \times n}, Y \in \mathbb{R}^{m \times n}, \bar{U} \in \mathbb{R}^{\overleftrightarrow{m} \times n}, \bar{V} \in \mathbb{R}^{\stackrel{\leftrightarrow}{m} \times n}$, $\bar{W} \in \mathbb{R}^{\overleftrightarrow{m} \times n}$, and the diagonal matrix $0<\bar{H} \in \mathbb{R}^{m \times m}$ such that for $\tau_{k}=0, \tau, \forall s \in\left[1,2^{m}\right], \forall l \in[1, \stackrel{\leftrightarrow}{m}]$, the following LMIs hold:

$$
\begin{aligned}
& \bar{\Lambda}_{1} \triangleq\left[\begin{array}{cc}
\hat{R}_{1} & \bar{M}_{1} \\
\bar{M}_{1}^{T} & \hat{R}_{1}
\end{array}\right]>0, \bar{\Lambda}_{2} \triangleq\left[\begin{array}{cc}
\bar{R}_{21} & \bar{M}_{2} \\
\bar{M}_{2}^{T} & \bar{R}_{21}
\end{array}\right]>0, \\
& \bar{\Xi}_{1}\left(\tau_{k}, s\right) \triangleq \Gamma_{1}^{T} \bar{P}_{1} \Gamma_{1}-\lambda_{1} \Gamma_{2}^{T} \bar{P}_{1} \Gamma_{2}-\lambda_{1}^{\tau} \Phi_{1}^{T} \bar{\Lambda}_{1} \Phi_{1} \\
& \quad-\Phi_{2}^{T}\left(\bar{Z}_{1} / \tilde{\sigma}_{1}\right) \Phi_{2}+\operatorname{Sym}\left(\bar{T}_{1} \bar{\Sigma}_{1}\right)+\bar{\Psi}_{1}<0, \\
& \bar{\Xi}_{2} \triangleq \Gamma_{3}^{T} \bar{P}_{2} \Gamma_{3}-\lambda_{2}\left(\Gamma_{4}^{T} \bar{P}_{2} \Gamma_{4}+\Phi_{3}^{T} \bar{\Lambda}_{2} \Phi_{3}+\Phi_{4}^{T} \hat{R}_{22}^{\tau} \Phi_{4}\right) \\
& \quad-\Phi_{5}^{T}\left(\bar{Z}_{2} / \tilde{\sigma}_{2}\right) \Phi_{5}+\operatorname{Sym}\left(\bar{T}_{2} \bar{\Sigma}_{2}+\bar{T}_{4} \bar{\Sigma}_{4}\right)+\bar{\Psi}_{2}<0, \\
& \bar{\Xi}_{3} \triangleq \Gamma_{5}^{T} \bar{P}_{2} \Gamma_{5}-\lambda_{2} \Gamma_{6}^{T} \bar{P}_{2} \Gamma_{6}-\lambda_{2} \Phi_{6}^{T} \hat{R}_{2}^{\tau} \Phi_{6} \\
& \quad-\Phi_{7}^{T}\left(\bar{Z}_{2} / \tilde{\sigma}_{2}\right) \Phi_{7}+\operatorname{Sym}\left(\bar{T}_{3} \bar{\Sigma}_{3}\right)+\bar{\Psi}_{3}<0, \\
& \left\{\begin{array}{c}
\bar{P}_{1} \leq \nu \bar{P}_{2}, \bar{Q}_{1} \leq \nu \bar{Q}_{2}, \bar{S}_{1 j} \leq \nu \bar{S}_{2 j}, \\
\bar{R}_{1 j} \leq \nu \bar{R}_{2 j}(j=1,2), \bar{Z}_{1} \leq \nu \bar{Z}_{2},
\end{array}\right. \\
& \bar{\Xi}_{4}(l) \triangleq\left[\begin{array}{cc}
1 /\left(\nu \lambda_{2}^{k^{*}}\right) & \bar{N}_{(l)} \\
\bar{N}_{(l)}^{T} & \operatorname{diag}\left\{\bar{P}_{1}, 0\right\}+\bar{\Psi}_{4} / \lambda_{1}
\end{array}\right] \geq 0
\end{aligned}
$$

where $\bar{N}_{(l)}=\left[\begin{array}{llll}\bar{U}_{(l)} & 0 & \bar{W}_{(l)} & \bar{V}_{(l)}\end{array}\right]$ and

$$
\begin{aligned}
& \bar{\Psi}_{1}=\operatorname{diag}\left\{\bar{Q}_{1}+\kappa \bar{S}_{11}+\sigma \bar{S}_{12}, 0,-\lambda_{1}^{\tau} \bar{Q}_{1}, 0,\right. \\
& \left.0,-\bar{S}_{11} / \tilde{\kappa}_{1},-\bar{S}_{12} / \tilde{\sigma}_{1}, \tau^{2} \bar{R}_{1}+\sigma \bar{Z}_{1}\right\}, \\
& \bar{\Psi}_{2}=\operatorname{diag}\left\{\bar{Q}_{2}+\kappa \bar{S}_{21}+\sigma \bar{S}_{22}, 0,-\lambda_{2}^{\tau} \bar{Q}_{2}, 0,\right. \\
& \left.-\bar{S}_{21} / \tilde{\kappa}_{2},-\bar{S}_{22} / \tilde{\sigma}_{2}, \tau^{2} \bar{R}_{2}+\sigma \bar{Z}_{2}, 0\right\}, \\
& \bar{\Psi}_{3}=\operatorname{diag}\left\{\bar{Q}_{2}+\kappa \bar{S}_{21}+\sigma \bar{S}_{22},-\lambda_{2}^{\tau} \bar{Q}_{2}, 0,\right. \\
& \left.-\bar{S}_{21} / \tilde{\kappa}_{2},-\bar{S}_{22} / \tilde{\sigma}_{2}, \tau^{2} \bar{R}_{2}+\sigma \bar{Z}_{2}\right\}, \\
& \bar{\Psi}_{4}=\operatorname{diag}\left\{0,\left(\lambda_{1}^{\tau} / \tau\right) \bar{Q}_{1}, \bar{S}_{12} / \tilde{\sigma}_{1}, \bar{S}_{11} / \tilde{\kappa}_{1}\right\} \\
& +\left(2 \lambda_{1}^{\tau} / \tilde{\tau}\right) \Phi_{8}^{T} \bar{R}_{1} \Phi_{8}+\left(1 / \tilde{\sigma}_{1}\right) \Phi_{9} \bar{Z}_{1} \Phi_{9}, \\
& \bar{T}_{1}=\left[\begin{array}{lll}
I & 0_{n \times 6 n} & \delta_{1} I
\end{array}\right]^{T}, \bar{T}_{3}=\left[\begin{array}{lll}
I & 0_{n \times 4 n} & \delta_{3} I
\end{array}\right]^{T}, \\
& \bar{T}_{2}=\left[\begin{array}{llll}
I & 0_{n \times 5 n} & \delta_{2} I & 0_{n \times m}
\end{array}\right]^{T}, \bar{T}_{4}=\left[\begin{array}{lll}
0_{m \times 7 n} & I
\end{array}\right]^{T} \text {, } \\
& \bar{\Sigma}_{1}=\left[(A-I) X^{T}+B \mathcal{D}_{s}^{-} \bar{U} \quad B D_{s} Y \quad 0_{n \times 3 n}\right. \\
& \left.A_{d} X^{T}+B \mathcal{D}_{s}^{-} \bar{V} \quad B \mathcal{D}_{s}^{-} \bar{W}-X^{T}\right] \text {, } \\
& \bar{\Sigma}_{2}=\left[\begin{array}{lllllll}
(A-I) X^{T} & B Y & 0 & 0 & A_{d} X^{T} & 0 & -X^{T}-B \bar{H}
\end{array}\right], \\
& \bar{\Sigma}_{3}=\left[\begin{array}{lllll}
(A-I) X^{T} & 0_{n \times 2 n} & A_{d} X^{T} & 0_{n \times n} & -X^{T}
\end{array}\right], \\
& \bar{\Sigma}_{4}=\left[\begin{array}{llll}
0_{m \times n} & Y & 0_{m \times 5 n} & -\bar{H}
\end{array}\right], \hat{R}_{1} \triangleq \operatorname{diag}\left\{\bar{R}_{1}, 3 \bar{R}_{1}\right\}, \\
& \hat{R}_{2}^{\tau} \triangleq \operatorname{diag}\left\{\bar{R}_{2}, 3 \varphi_{\tau} \bar{R}_{2}\right\}, \hat{R}_{22}^{\tau} \triangleq \operatorname{diag}\left\{\bar{R}_{22}, 3 \varphi_{\tau} \bar{R}_{22}\right\} \\
& \left(\varphi_{\tau} \triangleq(\tau+1) /(\tau-1)(\tau>1), \varphi_{1} \triangleq 1\right) .
\end{aligned}
$$

Then, for any $\phi(k) \in \mathscr{X}_{\rho}$ satisfying $V_{2}(0) \leq 1$, the system (1) can be exponentially stabilized by the controller (5) with $K=Y X^{-T}$.

Proof: If the LMIs (40)-(42) are feasible, it can be seen that the matrix $X$ is invertible. Then, we can define

$$
\left\{\begin{array}{l}
P_{\alpha} \triangleq \check{X}^{-1} \bar{P}_{\alpha} \check{X}^{-T}(\check{X} \triangleq \operatorname{diag}\{X, X, X\}) \\
Q_{\alpha} \triangleq X^{-1} \bar{Q}_{\alpha} X^{-T}, S_{\alpha j} \triangleq X^{-1} \bar{S}_{\alpha j} X^{-T} \\
R_{\alpha j} \triangleq X^{-1} \bar{R}_{\alpha j} X^{-T}, Z_{\alpha} \triangleq X^{-1} \bar{Z}_{\alpha} X^{-T} \\
T_{i 1} \triangleq X^{-1}, T_{i 2} \triangleq \delta_{i} X^{-1}, \alpha, j=1,2, i=1,2,3 \\
M_{1} \triangleq \hat{X}^{-1} \bar{M}_{1} \hat{X}^{-T}(\hat{X} \triangleq \operatorname{diag}\{X, X\}) \\
M_{2} \triangleq X^{-1} \bar{M}_{2} X^{-T}, H \triangleq \bar{H}^{-T}, K \triangleq Y X^{-T} \\
U \triangleq \bar{U} X^{-T}, V \triangleq \bar{V} X^{-T}, W \triangleq \bar{W} X^{-T}
\end{array}\right.
$$

Performing some congruence transformations to LMIs (39)-(44) (see [2], [28]), and using the notations in (45), the matrix inequalities (14)-(19) in Theorem 1 can be, respectively, obtained.

If the input delay $\tau_{k}$ is not incorporated in the system (1), the corresponding closed-loop system can be written as

$$
\begin{aligned}
x(k+1)= & \sum_{s=1}^{2^{m}} \varpi_{s}^{k}\left\{\left[A+B\left(D_{s} K+\mathcal{D}_{s}^{-} U\right)\right] x(k)\right. \\
& +\left(A_{d}+B \mathcal{D}_{s}^{-} V\right) \sum_{i=1}^{+\infty} \mu_{i} x(k-i) \\
& \left.+B \mathcal{D}_{s}^{-} W \sum_{j=1}^{+\infty} \mu_{j} \sum_{i=k-j}^{k-1} x(i)\right\}, k \geq 0 .
\end{aligned}
$$

Choose the following augmented Lyapunov functional:

$$
\begin{aligned}
\hat{V}(k) & =\hat{\eta}^{T}(k) \operatorname{diag}\left\{X^{-1}, X^{-1}\right\} \bar{P} \operatorname{diag}\left\{X^{-T}, X^{-T}\right\} \hat{\eta}(k) \\
+ & \sum_{j=1}^{+\infty} \mu_{j} \sum_{i=k-j}^{k-1} \lambda_{1}^{k-i-1} x^{T}(i) X^{-1} \bar{S}_{1} X^{-T} x(i) \\
& +\sum_{l=1}^{+\infty} \mu_{l} \sum_{j=1}^{l} \sum_{i=k-j}^{k-1} \lambda_{1}^{k-i-1} x^{T}(i) X^{-1} \bar{S}_{2} X^{-T} x(i) \\
& +\sum_{l=1}^{+\infty} \mu_{l} \sum_{j=1}^{l} \sum_{i=k-j}^{k-1} \lambda_{1}^{k-i-1} y^{T}(i) X^{-1} \bar{Z} X^{-T} y(i)
\end{aligned}
$$

where $\hat{\eta}(k)=\left[\begin{array}{ll}x^{T}(k) & \sum_{j=1}^{+\infty} \mu_{j} \sum_{i=k-j}^{k-1} x^{T}(i)\end{array}\right]^{T}$. Then, it is easy to obtain the following control design condition.

Corollary 1: Let the scalars $0<\lambda_{1} \leq 1$ and $\delta \neq 0$ be given. Assume that there exist matrices $0<\bar{P} \in \mathbb{R}^{2 n \times 2 n}, 0<\bar{S}_{1} \in \mathbb{R}^{n \times n}$, $0<\bar{S}_{2} \in \mathbb{R}^{n \times n}, 0<\bar{Z} \in \mathbb{R}^{n \times n}, X \in \mathbb{R}^{n \times n}, Y \in \mathbb{R}^{m \times n}$, $\bar{U} \in \mathbb{R}^{\stackrel{\leftrightarrow}{m} \times n}, \bar{V} \in \mathbb{R}^{\overleftrightarrow{m} \times n}, \bar{W} \in \mathbb{R}^{\stackrel{\leftrightarrow}{m} \times n}$, such that for $\forall s \in\left[1,2^{m}\right]$, $\forall l \in[1, \stackrel{\leftrightarrow}{m}]$, the following LMIs are feasible:

$$
\begin{aligned}
\hat{\Xi}_{1}(s) \triangleq & \hat{\Gamma}_{1}^{T} \bar{P} \hat{\Gamma}_{1}-\lambda_{1} \hat{\Gamma}_{2}^{T} \bar{P} \hat{\Gamma}_{2}+\hat{\Psi}_{1} \\
& -\hat{\Phi}_{1}^{T}\left(\bar{Z} / \tilde{\sigma}_{1}\right) \hat{\Phi}_{1}+\operatorname{Sym}\left(\hat{T}_{1} \hat{\Sigma}_{1}\right)<0, \\
\hat{\Xi}_{2}(l) \triangleq & {\left[\begin{array}{cc}
1 & \hat{N}_{(l)} \\
\hat{N}_{(l)}^{T} & \operatorname{diag}\{\bar{P}, 0\}+\hat{\Psi}_{2} / \lambda_{1}
\end{array}\right] \geq 0 }
\end{aligned}
$$

where $\hat{N}_{(l)}=\left[\begin{array}{lll}\bar{U}_{(l)} & \bar{W}_{(l)} & \bar{V}_{(l)}\end{array}\right]$ and

$$
\begin{aligned}
& \hat{\Gamma}_{1}=\left[\begin{array}{cccc}
I & 0 & 0 & I \\
\kappa I & -I & I & 0
\end{array}\right], \hat{\Gamma}_{2}=\left[\begin{array}{cccc}
I & 0 & 0 & 0 \\
0 & 0 & I & 0
\end{array}\right] \text {, } \\
& \hat{\Phi}_{1}=\left[\begin{array}{lll}
\kappa I & -I & 0_{n \times 2 n}
\end{array}\right], \hat{T}_{1}=\left[\begin{array}{lll}
I & 0_{n \times 2 n} & \delta I
\end{array}\right]^{T}, \\
& \hat{\Sigma}_{1}=\left[(A-I) X^{T}+B\left(\mathcal{D}_{s}^{-} \bar{U}+D_{s} Y\right)\right. \\
& \left.A_{d} X^{T}+B \mathcal{D}_{s}^{-} \bar{V} \quad B \mathcal{D}_{s}^{-} \bar{W}-X^{T}\right] \text {, } \\
& \hat{\Psi}_{1}=\operatorname{diag}\left\{\kappa \bar{S}_{1}+\sigma \bar{S}_{2},-\bar{S}_{1} / \tilde{\kappa}_{1},-\bar{S}_{2} / \tilde{\sigma}_{1}, \sigma \bar{Z}\right\}, \\
& \hat{\Psi}_{2}=\operatorname{diag}\left\{0, \bar{S}_{2} / \tilde{\sigma}_{1}, \bar{S}_{1} / \tilde{\kappa}_{1}\right\}+\left(1 / \tilde{\sigma}_{1}\right) \\
& \times\left[\begin{array}{lll}
\kappa I & 0 & -I
\end{array}\right]^{T} \bar{Z}\left[\begin{array}{lll}
\kappa I & 0 & -I
\end{array}\right] .
\end{aligned}
$$

Then, for any $\phi(k) \in \mathscr{X}_{\rho}$ satisfying $\hat{V}_{1}(0) \leq 1$, the system (1) can be exponentially stabilized by (5) with $K=Y X^{-T}$.

Remark 5: In recent years, the local stabilization problem has been sufficiently addressed for discrete-time systems with time delays under saturating actuators [2], [16], [17], [19]. Different from such recent results where a single discrete state delay is involved, our obtained results are concerned with the discrete-time systems with both distributed state delay and fast-varying input delays. In fact, distributed delays are often encountered in various applications such as engineering systems, traffic flow models, biological systems and neural networks [4], [18], [22]. Moreover, almost all practical control 
systems are subject to input delays (e.g., actuator and transmission delays). The results presented in Theorem 2 and Corollary 1 serve as indispensable complements of the existing results. For systems with finite distributed delay $\sum_{i=1}^{h} \mu_{i} x(k-i)$, the corresponding results can be readily obtained by revising the notations of $\kappa, \sigma, \tilde{\kappa}_{\alpha}$ and $\tilde{\sigma}_{\alpha}$.

For the case that the distributed state delay is not involved in (1), one can select the following Lyapunov functional:

$$
\check{V}(k)=\left\{\begin{array}{l}
\check{V}_{1}(k), k \in\left[k^{*},+\infty\right), \\
\check{V}_{2}(k), k \in\left[0, k^{*}\right)
\end{array}\right.
$$

where

$$
\begin{aligned}
\check{V}_{\alpha}(k)= & \check{\eta}^{T}(k) \operatorname{diag}\left\{X^{-1}, X^{-1}\right\} \bar{P}_{\alpha} \operatorname{diag}\left\{X^{-T}, X^{-T}\right\} \check{\eta}(k) \\
& +\sum_{i=k-\tau}^{k-1} \lambda_{\alpha}^{k-i-1} x^{T}(i) X^{-1} \bar{Q}_{\alpha} X^{-T} x(i) \\
& +\tau \sum_{j=-\tau}^{-1} \sum_{i=k+j}^{k-1} \lambda_{\alpha}^{k-i-1} y^{T}(i) X^{-1} \bar{R}_{\alpha} X^{-T} y(i)
\end{aligned}
$$

with $\check{\eta}(k)=\left[\begin{array}{ll}x^{T}(k) & \sum_{i=k-\tau}^{k-1} x^{T}(i)\end{array}\right]^{T}$ and $\bar{R}_{\alpha}=\bar{R}_{\alpha 1}+\bar{R}_{\alpha 2}$. Then, the following sufficient condition is readily obtained.

Corollary 2: Let the scalars $0<\lambda_{1} \leq 1, \lambda_{2}>1, \nu>0, \delta_{i} \neq 0$ ( $i=1,2,3)$ and the integer $k^{*} \geq 1$ be given. Assume that there exist matrices $0<\bar{P}_{\alpha} \in \mathbb{R}^{2 n \times 2 n}, 0<\bar{Q}_{\alpha} \in \mathbb{R}^{n \times n}, 0<\bar{R}_{\alpha j} \in \mathbb{R}^{n \times n}$ $(\alpha, j=1,2), \bar{M}_{1} \in \mathbb{R}^{2 n \times 2 n}, \bar{M}_{2} \in \mathbb{R}^{n \times n}, X \in \mathbb{R}^{n \times n}, Y \in \mathbb{R}^{m \times n}$, $\bar{U} \in \mathbb{R}^{\overleftrightarrow{m} \times n}$, and the diagonal matrix $0<\bar{H} \in \mathbb{R}^{m \times m}$ such that, for $\tau_{k}=0, \tau, \forall s \in\left[1,2^{m}\right], \forall l \in[1, \overleftrightarrow{m}]$, the LMIs (39) and

$$
\begin{aligned}
& \check{\Xi}_{1}\left(\tau_{k}, s\right) \triangleq \check{\Gamma}_{1}^{T} \bar{P}_{1} \check{\Gamma}_{1}-\lambda_{1} \check{\Gamma}_{2}^{T} \bar{P}_{1} \check{\Gamma}_{2}+\check{\Psi}_{1} \\
& -\lambda_{1}^{\tau} \check{\Phi}_{1}^{T} \bar{\Lambda}_{1} \check{\Phi}_{1}+\operatorname{Sym}\left(\check{T}_{1} \check{\Sigma}_{1}\right)<0, \\
& \check{\Xi}_{2} \triangleq \check{\Gamma}_{3}^{T} \bar{P}_{2} \check{\Gamma}_{3}-\lambda_{2}\left(\check{\Gamma}_{4}^{T} \bar{P}_{2} \breve{\Gamma}_{4}+\check{\Phi}_{2}^{T} \bar{\Lambda}_{2} \check{\Phi}_{2}\right)+\check{\Psi}_{2} \\
& -\lambda_{2} \check{\Phi}_{3}^{T} \hat{R}_{22}^{\tau} \check{\Phi}_{3}+\operatorname{Sym}\left(\check{T}_{2} \check{\Sigma}_{2}+\check{T}_{4} \check{\Sigma}_{4}\right)<0, \\
& \check{\Xi}_{3} \triangleq \check{\Gamma}_{5}^{T} \bar{P}_{2} \check{\Gamma}_{5}-\lambda_{2} \check{\Gamma}_{6}^{T} \bar{P}_{2} \check{\Gamma}_{6}+\check{\Psi}_{3} \\
& -\lambda_{2} \check{\Phi}_{4}^{T} \hat{R}_{2}^{\tau} \check{\Phi}_{4}+\operatorname{Sym}\left(\check{T}_{3} \check{\Sigma}_{3}\right)<0, \\
& \bar{P}_{1} \leq \nu \bar{P}_{2}, \bar{Q}_{1} \leq \nu \bar{Q}_{2}, \bar{R}_{1 j} \leq \nu \bar{R}_{2 j}, j=1,2 \text {, }
\end{aligned}
$$

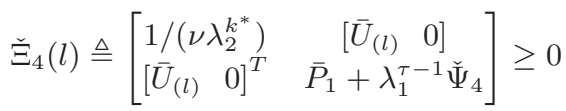

hold, where $\hat{R}_{1}, \hat{R}_{2}^{\tau}, \hat{R}_{22}^{\tau}$ are denoted in Theorem 2 and

$$
\begin{aligned}
& \check{\Gamma}_{1}=\left[\begin{array}{cccccc}
I & 0 & 0 & 0 & 0 & I \\
0 & -I & -I & \check{\tau}_{k} I & \hat{\tau}_{k} I & 0
\end{array}\right] \text {, } \\
& \check{\Gamma}_{2}=\left[\begin{array}{cccccc}
I & 0 & 0 & 0 & 0 & 0 \\
-I & -I & 0 & \check{\tau}_{k} I & \hat{\tau}_{k} I & 0
\end{array}\right] \text {, } \\
& \check{\Gamma}_{3}=\left[\begin{array}{cccccc}
I & 0 & 0 & 0 & I & 0 \\
0 & 0 & -I & \tilde{\tau} I & 0 & 0
\end{array}\right], \\
& \check{\Gamma}_{4}=\left[\begin{array}{ccccc}
I & 0 & 0 & 0 & 0_{n \times(n+m)} \\
-I & 0 & 0 & \tilde{\tau} I & 0_{n \times(n+m)}
\end{array}\right] \text {, } \\
& \check{\Gamma}_{5}=\left[\begin{array}{cccc}
I & 0 & 0 & I \\
0 & -I & \tilde{\tau} I & 0
\end{array}\right], \check{\Gamma}_{6}=\left[\begin{array}{cccc}
I & 0 & 0 & 0 \\
-I & 0 & \tilde{\tau} I & 0
\end{array}\right] \text {, } \\
& \check{\Phi}_{1}=\left[\begin{array}{cccccc}
I & -I & 0 & 0 & 0 & 0 \\
I & I & 0 & -2 I & 0 & 0 \\
0 & I & -I & 0 & 0 & 0 \\
0 & I & I & 0 & -2 I & 0
\end{array}\right] \text {, } \\
& \check{\Phi}_{2}=\left[\begin{array}{cccc}
I & -I & 0 & 0_{n \times(2 n+m)} \\
0 & I & -I & 0_{n \times(2 n+m)}
\end{array}\right] \text {, } \\
& \check{\Phi}_{3}=\left[\begin{array}{ccccc}
I & 0 & -I & 0 & 0_{n \times(n+m)} \\
I & 0 & I & -2 I & 0_{n \times(n+m)}
\end{array}\right],
\end{aligned}
$$

$$
\begin{aligned}
& \check{\Phi}_{4}=\left[\begin{array}{cccc}
I & -I & 0 & 0 \\
I & I & -2 I & 0
\end{array}\right], \check{\Phi}_{5}=\left[\begin{array}{ll}
\tau I & -I
\end{array}\right], \\
& \check{\Psi}_{1}=\operatorname{diag}\left\{\bar{Q}_{1}, 0,-\lambda_{1}^{\tau} \bar{Q}_{1}, 0,0, \tau^{2} \bar{R}_{1}\right\}, \\
& \check{\Psi}_{2}=\operatorname{diag}\left\{\bar{Q}_{2}, 0,-\lambda_{2}^{\tau} \bar{Q}_{2}, 0, \tau^{2} \bar{R}_{2}, 0\right\} \text {, } \\
& \check{\Psi}_{3}=\operatorname{diag}\left\{\bar{Q}_{2},-\lambda_{2}^{\tau} \bar{Q}_{2}, 0, \tau^{2} \bar{R}_{2}\right\}, \\
& \check{\Psi}_{4}=\operatorname{diag}\left\{0, \bar{Q}_{1} / \tau\right\}+(2 / \tilde{\tau}) \check{\Phi}_{5}^{T} \bar{R}_{1} \check{\Phi}_{5}, \\
& \bar{\Psi}_{4}=\operatorname{diag}\left\{0, \lambda_{1}^{\tau} \bar{Q}_{1} / \tau, \bar{S}_{12} / \tilde{\sigma}_{1}, \bar{S}_{11} / \tilde{\kappa}_{1}\right\} \\
& +\left(2 \lambda_{1}^{\tau} / \tilde{\tau}\right) \Phi_{8}^{T} \bar{R}_{1} \Phi_{8}+\left(1 / \tilde{\sigma}_{1}\right) \Phi_{9} \bar{Z}_{1} \Phi_{9}, \\
& \check{T}_{1}=\left[\begin{array}{lll}
I & 0_{n \times 4 n} & \delta_{1} I
\end{array}\right]^{T}, \check{T}_{3}=\left[\begin{array}{lll}
I & 0_{n \times 2 n} & \delta_{3} I
\end{array}\right]^{T} \text {, } \\
& \check{T}_{2}=\left[\begin{array}{llll}
I & 0_{n \times 3 n} & \delta_{2} I & 0
\end{array}\right]^{T}, \check{T}_{4}=\left[\begin{array}{lll}
0_{m \times 5 n} & I
\end{array}\right]^{T} \text {, } \\
& \check{\Sigma}_{1}=\left[(A-I) X^{T}+B \mathcal{D}_{s}^{-} \bar{U} \quad B D_{s} Y \quad 0_{n \times 3 n}\right. \\
& \left.-X^{T}\right], \check{\Sigma}_{4}=\left[\begin{array}{llll}
0_{m \times n} & Y & 0_{m \times 3 n} & -\bar{H}
\end{array}\right], \\
& \check{\Sigma}_{2}=\left[\begin{array}{lllll}
(A-I) X^{T} & B Y & 0_{n \times 2 n} & -X^{T} & -B \bar{H}
\end{array}\right], \\
& \check{\Sigma}_{3}=\left[\begin{array}{lll}
(A-I) X^{T} & 0_{n \times 2 n} & -X^{T}
\end{array}\right] .
\end{aligned}
$$

Then, for any $\phi(k) \in \mathscr{X}_{\rho}$ satisfying $\check{V}_{2}(0) \leq 1$, the system (1) can be exponentially stabilized by (5) with $K=Y X^{-T}$.

Remark 6: Recently, the local stabilization problem has been studied in [9] for linear input-delay systems with saturating actuators. Unlike [9], the constraint on the time-varying input delay is removed in this paper. Furthermore, the analysis approach proposed in [9] is no longer applicable because of the existence of multiple intervals with zero control signal and two dynamics within the interval $\left[0, k^{*}\right)$. In this paper, the more flexible piecewise Lyapunov functional (50) is proposed to characterize the possible state evolutions of different dynamics within the whole time interval. Moreover, different from the techniques used in [9], the augmented Lyapunov functional, the Wirtinger-based inequality and the current-state-dependent polytopic model are utilized together in this paper to reduce the conservatism.

Remark 7: In case the saturation level is non-unity, that is, $\operatorname{sat}\left(u_{l}\right)$ $=\operatorname{sgn}\left(u_{l}\right) \min \left\{\left|u_{l}\right|, \bar{u}_{l}\right\}$, the matrices $B$ and $Y$ in Theorem 2 and

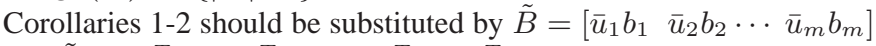
and $\tilde{Y}=\left[\begin{array}{lll}y_{1}^{T} / \bar{u}_{1} & y_{2}^{T} / \bar{u}_{2} \cdots y_{m}^{T} / \bar{u}_{m}\end{array}\right]^{T}$, respectively, where $b_{l}$ is the $l$-th column of $B$ and $y_{l}$ is the $l$-th row of $Y$. Also, if the integer $k^{*}$ is unknown, $k^{*}$ in LMIs (44) and (55) should be replaced by $\tau$.

In the subsequent part, we will address the maximization problem of the initial condition set $\mathscr{X}_{\rho}$. First, let us introduce the LMI

$$
\bar{P}_{2} \leq \operatorname{diag}\left\{\mathcal{J}_{1}, \mathcal{J}_{2}, \mathcal{J}_{3}\right\}\left(\mathcal{J}_{i}>0, i=1,2,3\right) .
$$

Using (56) and Lemma 2, and noting (45), it follows that

$$
\begin{aligned}
& V_{2}(0) \leq\left[\lambda_{M}\left(X^{-1} \mathcal{J}_{1} X^{-T}\right)+\tau^{2} \lambda_{M}\left(X^{-1} \mathcal{J}_{2} X^{-T}\right)\right. \\
& +\sigma^{2} \lambda_{M}\left(X^{-1} \mathcal{J}_{3} X^{-T}\right)+\varphi_{1} \lambda_{M}\left(X^{-1} \bar{Q}_{2} X^{-T}\right) \\
& \left.+\varphi_{22} \lambda_{M}\left(X^{-1} \bar{S}_{21} X^{-T}\right)+\varphi_{32} \lambda_{M}\left(X^{-1} \bar{S}_{22} X^{-T}\right)\right] \rho_{1}^{2} \\
& +\left[\varphi_{4} \lambda_{M}\left(X^{-1} \bar{R}_{2} X^{-T}\right)+\varphi_{32} \lambda_{M}\left(X^{-1} \bar{Z}_{2} X^{-T}\right)\right] \rho_{2}^{2}
\end{aligned}
$$

To estimate $\hat{V}(0)$ in Corollary 1 , we introduce the LMI

$$
\bar{P} \leq \operatorname{diag}\left\{\mathcal{J}_{1}, \mathcal{J}_{2}\right\}\left(\mathcal{J}_{j}>0, j=1,2\right) .
$$

Correspondingly, $\hat{V}(0)$ can be enlarged as follows:

$$
\begin{aligned}
\hat{V}(0) \leq & {\left[\lambda_{M}\left(X^{-1} \mathcal{J}_{1} X^{-T}\right)+\sigma^{2} \lambda_{M}\left(X^{-1} \mathcal{J}_{2} X^{-T}\right)\right.} \\
& \left.+\varphi_{21} \lambda_{M}\left(X^{-1} \bar{S}_{1} X^{-T}\right)+\varphi_{31} \lambda_{M}\left(X^{-1} \bar{S}_{2} X^{-T}\right)\right] \\
& \times \rho_{1}^{2}+\varphi_{31} \lambda_{M}\left(X^{-1} \bar{Z} X^{-T}\right) \rho_{2}^{2}
\end{aligned}
$$

Next, we introduce the matrix inequality $X^{-1} X^{-T} \leq x I(x>0$ is a scalar) [1], which can be guaranteed by the following LMI:

$$
\left[\begin{array}{cc}
x I & I \\
I & X+X^{T}-I
\end{array}\right] \geq 0 .
$$


Moreover, we introduce the following two sets of LMIs:

$$
\begin{aligned}
& \left\{\begin{array}{l}
\mathcal{J}_{i} \leq p_{i} I, i=1,2,3, \bar{Q}_{2} \leq q I, \\
\bar{S}_{2 j} \leq s_{j} I, \bar{R}_{2 j} \leq r_{j} I, j=1,2, \bar{Z}_{2} \leq z I,
\end{array}\right. \\
& \mathcal{J}_{j} \leq p_{j} I, \bar{S}_{j} \leq s_{j} I, i=1,2, \bar{Z} \leq z I .
\end{aligned}
$$

Then, it is seen that the maximization of the admissible initial condition set $\mathscr{X}_{\rho}$ in Theorem 2 and Corollary 1 can be, respectively, formulated by the following convex optimization problems:

$$
\begin{aligned}
& \text { Prob.1. } \min _{\bar{P}_{\alpha}, \bar{Q}_{\alpha}, \bar{S}_{\alpha j}, \bar{R}_{\alpha j}, \bar{Z}_{\alpha}, \bar{M}_{\alpha}, X, Y, \bar{U}, \bar{V}, \bar{W}, \bar{H}, \mathcal{J}_{i}, x, p_{i}, q, s_{j}, r_{j}, z} \chi_{1}, \\
& \text { s.t., LMIs in Theorem } 2 \text { and LMIs (56), (60), (61) hold, } \\
& \text { Prob.2. } \min _{\bar{P}_{\alpha}, \bar{S}_{\alpha j}, \bar{Z}_{\alpha}, X, Y, \bar{U}, \bar{V}, \bar{W}, \mathcal{J}_{j}, x, p_{j}, s_{j}, z} \chi_{2} \text {, s.t., }
\end{aligned}
$$

LMIs in Corollary 1 and LMIs (58), (60), (62) hold

where $\chi_{1}=\epsilon x+p_{1}+\tau^{2} p_{2}+\sigma^{2} p_{3}+\varphi_{1} q+\varphi_{22} s_{1}+\varphi_{32} s_{2}+\varphi_{4} r_{1}+$ $\varphi_{4} r_{2}+\varphi_{32} z$ and $\chi_{2}=\epsilon x+p_{1}+\sigma^{2} p_{2}+\varphi_{21} s_{1}+\varphi_{31} s_{2}+\varphi_{31} z$ (the scalar $\epsilon>0$ is a weighting parameter).

Remark 8: In this paper, our obtained results are based on the novel polytopic models. If the same research is performed by using traditional polytopic model, the corresponding optimization problems are directly obtained by setting $\bar{V}=\bar{W}=0$ in (40) and (48), which are referred to as Prob.1' and Prob.2', respectively, in this paper.

For the case that the system (1) has a single input delay where $u(k)=0$ for $k<0$, we can see that the solution of (1) does not depend on the values of $x(k)$ for $k \leq 0$ [9]. Then, we can define $x(k)=\phi(k)=x_{0},-\tau \leq k \leq 0$. In this case, we assume that the initial condition $x_{0}$ belongs to an ellipsoid as follows [9]:

$$
\mathscr{E} \triangleq\left\{x_{0} \in \mathbb{R}^{n}: x_{0}^{T} P x_{0} \leq 1\right\} .
$$

From (50), it is seen that $\check{V}_{2}(0)$ can be written as

$$
\check{V}_{2}(0)=x_{0}^{T} X^{-1} \tilde{P} X^{-T} x_{0} \triangleq x_{0}^{T} P x_{0} .
$$

where $\tilde{P}=\left[\begin{array}{ll}I & \tau I\end{array}\right] \bar{P}_{2}\left[\begin{array}{ll}I & \tau I\end{array}\right]^{T}+\varphi_{1} \bar{Q}_{2}$. Let the LMI

$$
\tilde{P}=\left[\begin{array}{ll}
I & \tau I
\end{array}\right] \bar{P}_{2}\left[\begin{array}{ll}
I & \tau I
\end{array}\right]^{T}+\varphi_{1} \bar{Q}_{2} \leq p I
$$

be given. Then, the maximization of the admissible initial condition set $\mathscr{E}$ involving in Corollary 2 can be described as follows:

$$
\text { Prob.3. } \min _{\bar{P}_{\alpha}, \bar{Q}_{\alpha}, \bar{R}_{\alpha}, \bar{M}_{\alpha}, X, Y, \bar{U}, \bar{H}, x, p} \epsilon x+p \text {, s.t., }
$$

LMIs in Corollary 2 and LMIs (60), (65) hold.

\section{NUMERICAL EXAMPLES}

Example 1: Consider the system described by (1) where

$$
\begin{aligned}
& A=\left[\begin{array}{cc}
1.1 & 0.15 \\
0.03 & 0.8
\end{array}\right], \quad A_{d}=\left[\begin{array}{cc}
0 & -0.1 \\
0 & 0
\end{array}\right], B=\left[\begin{array}{c}
1 \\
0.1
\end{array}\right], \\
& \mu^{i}=2^{-i}, \bar{u}_{1}=15, \quad 0 \leq \tau_{k}=2+(-1)^{k} \leq 3 .
\end{aligned}
$$

For this example, we first consider the special case without input delay. By solving Prob.2 and Prob.2' with $\delta=1.5$ and $\epsilon=2 \times 10^{9}$, the admissible bounds of the initial condition set $\mathscr{X}_{\rho}\left(\rho_{1}=\rho_{2} \triangleq \rho\right)$ can be readily obtained for different $\lambda_{1}(>1 / 2)$, which are given in Table I. Recalling that Prob.2' is based on the traditional polytopic model, Table I shows that our proposed distributed-delay-dependent polytopic model is really effective in reducing the conservatism.

Next, we consider the more general case. By solving Prob.1 with $\delta_{1}=\delta_{2}=\delta_{3}=4, \epsilon=2 \times 10^{9}, \lambda_{1}=1, \lambda_{2}=1.24$ and $\nu=$ 0.97 , it is found that the initial condition set $\mathscr{X}_{\rho}$ can be bounded by $2.0116 \rho_{1}^{2}+1.7704 \rho_{2}^{2} \leq 10^{4}$ with $K=\left[\begin{array}{lll}-0.1695 & -0.0397\end{array}\right]$. In particular, we have $\rho_{1} \leq 70.5065$ for the case $\rho_{2}=0$. Using the
TABLE I

The Admissible bounds $(\rho)$ OF The initial Condition Set $\mathscr{X}_{\rho}$

\begin{tabular}{cccccc}
\hline$\lambda_{1}$ & 0.90 & 0.93 & 0.95 & 0.87 & 1.0 \\
\hline Prob.2 & 74.3899 & 82.8034 & 89.5207 & 97.3417 & 110.5246 \\
\hline Prob. $^{\prime}$ & 60.1129 & 69.6121 & 76.7917 & 84.7264 & 98.1399 \\
\hline
\end{tabular}

TABLE II

THE ADMISSIBLE RADII ( $\check{\rho})$ OF THE INITIAL BALL

\begin{tabular}{cccccc}
\hline$\tau$ & 3 & 4 & 5 & 6 & 7 \\
\hline$[9]$ & 2.2616 & 1.9100 & 1.5745 & 1.2022 & infeasible \\
\hline Prob.3 & 2.5263 & 2.2977 & 2.0391 & 1.6340 & 0.9694 \\
\hline
\end{tabular}

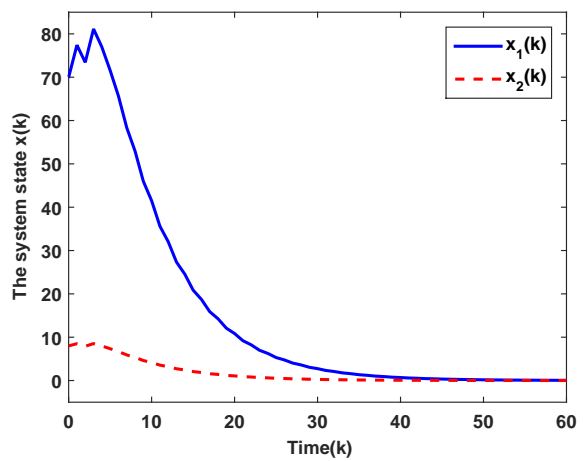

Fig. 1. The state evolutions of the closed-loop system.

above controller gain, the state evolution of this system is plotted in Fig. 1. In the simulation, the initial condition is selected as $\phi(k)=$ $\left[\begin{array}{ll}70 & 8\end{array}\right]^{T} \in \mathscr{X}_{\rho}$. Noting that the open-loop system is not stable, it is seen from Fig. 1 that our proposed control scheme is effective.

Example 2: Consider the discrete time-delay system (1) where

$$
\begin{aligned}
& A=\left[\begin{array}{cc}
1.11 & -0.06 \\
0.05 & 0.9
\end{array}\right], B=\left[\begin{array}{l}
0.1 \\
0.1
\end{array}\right], A_{d}=0, \\
& \bar{u}_{1}=5,0 \leq \tau_{k} \leq \tau, k^{*} \text { is unknown. }
\end{aligned}
$$

For this example, by solving the optimization problems in [9] and Prob.3 of this paper, we obtain some maximum admissible radii $\check{\rho} \triangleq$ $\sqrt{1 / \lambda_{M}(P)}$ of the initial ball contained in the region of attraction, which are listed in Table II. In particular, when $\tau=5$, we have

$$
\begin{aligned}
& \mathcal{X} \triangleq\left\{x_{0} \in \mathbb{R}^{2}: x_{0}^{T}\left[\begin{array}{cc}
0.3683 & -0.1136 \\
-0.1136 & 0.0350
\end{array}\right] x_{0} \leq 1\right\}([9]), \\
& \mathscr{E} \triangleq\left\{x_{0} \in \mathbb{R}^{2}: x_{0}^{T}\left[\begin{array}{cc}
0.2196 & -0.0677 \\
-0.0677 & 0.0209
\end{array}\right] x_{0} \leq 1\right\} \text { (Prob.3), } \\
& K=\left[\begin{array}{lll}
-2.1527 & 0.6683
\end{array}\right]([9]), K=[-2.13790 .6545] \text { (Prob.3). }
\end{aligned}
$$

In solving the optimization problem in [9], we select $\lambda=1, \mu=$ $1.3, \beta=1, \sigma=0.001$ and $\varepsilon=12$. In solving Prob.3, we choose $\delta_{1}=10, \delta_{2}=\delta_{3}=8, \lambda_{1}=1, \lambda_{2}=1.23, \nu=0.9$ and $\epsilon=100$. In Fig. 2, we plot part bounds of the sets $\mathcal{X}$ and $\mathscr{E}$ and some state trajectories of the closed-loop system. It is seen from Table II and Fig. 2 that our proposed result can provide a larger estimate of the region of attraction than that in [9]. Moreover, we notice that two trajectories starting not so far from the set $\mathscr{E}$ diverge. In addition, it is worth mentioning that our result removes the constraint on $\tau_{k}$.

\section{Conclusions}

In this paper, the local stabilization problem has been addressed for discrete-time systems with both distributed state delay and fast- 


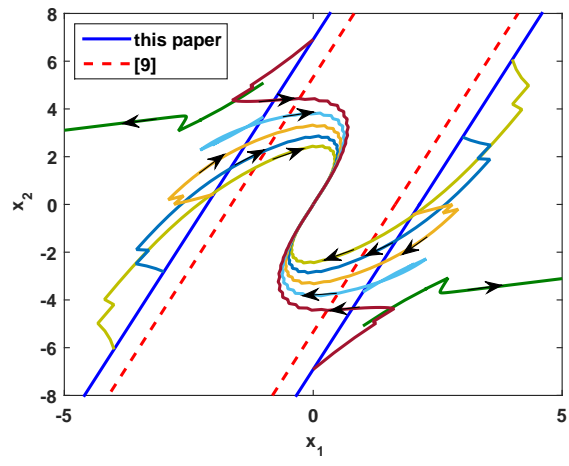

Fig. 2. Part bounds of the sets $\mathcal{X}$ and $\mathscr{E}$ and the state trajectories.

varying input delay under saturating actuators. By using a novel polytopic model, the piecewise Lyapunov functional and some summation inequalities, a local stabilization condition has been established in terms of LMIs. The special cases with either state delay or input delay have also been addressed. The proposed results in our paper can be easily extended to the case with discrete state delay. Also, it is interesting to consider the local stabilization problem for saturated systems with distributed input delay, which is our future work.

Here, it is worth mentioning that the techniques dealing with the time delays in this paper are somewhat conservative. By using the recent developed inequalities [11], [15], [26], [29], one can establish some more effective results, which is our further research. In addition, it is noted that the our results are based on the assumption that $u(k)=$ 0 for $k<0$. In case the past states can be used for feedback, the LMI $\bar{\Lambda}_{2}>0$ in (39), LMIs (41)-(43), LMIs (52)-(54) should be removed, the term $1 /\left(\nu \lambda_{2}^{k^{*}}\right)$ in LMIs (44) and (55) should be set as 1. Moreover, the constrains on $\phi(k)$ in Theorem 2 and Corollary 2 should be revised as $V_{1}(0) \leq 1$ and $\check{V}_{1}(0) \leq 1$, respectively.

\section{REFERENCES}

[1] Y. Chen, S. Fei, and Y. Li, "Robust stabilization for uncertain saturated time-delay systems: a distributed-delay-dependent polytopic approach," IEEE Trans. Autom. Control, vol. 62, no. 7, pp. 3455-3460, 2017.

[2] Y. Chen, Z. Wang, S. Fei, and Q.-L. Han, "Regional stabilization for discrete time-delay systems with actuator saturations via a delaydependent polytopic approach," IEEE Trans. Autom. Control, vol. 64, no. 3, pp. 1257-1264, 2019.

[3] Y. Chen, Z. Wang, Y. Liu, and F. E., Alsaadi, "Stochastic stability for distributed delay neural networks via augmented Lyapunov-Krasovskii functionals," Appl. Math. Comput., vol. 338, pp. 869-881, 2018.

[4] E. Fridman, Introduction to time-delay systems: Analysis and control. Basel, Switzerland: Birkhäuser, 2014

[5] E. Fridman, A. Pila, and U. Shaked, "Regional stabilization and $H_{\infty}$ control of time-delay systems with saturating actuators, Int. J. Robust Nonlin. Control," vol. 13, no. 9, pp. 885-907, 2003.

[6] J. M. Gomes da Silva Jr, and S. Tarbouriech, "Antiwindup design with guaranteed regions of stability: an LMI-based approach," IEEE Trans. Autom. Control, vol. 50, no. 1, pp. 106-111, 2005.

[7] Z. Lin, Low gain feedback. London, U.K.: Springer-Verlag, 1999.

[8] Z. Lin, and H. Fang, "On asymptotic stabilizability of linear systems with delayed input," IEEE Trans. Autom. Control, vol. 52, no. 6, pp. 998 1013, 2007.

[9] K. Liu, and E. Fridman, "Delay-dependent methods and the first delay interval," Syst. Control Lett., vol. 64, pp. 57-63, 2014.

[10] K. Liu, E. Fridman, K. H. Johansson, and Y. Xia, "Generalized Jensen inequalities with application to stability analysis of systems with distributed delays over infinite time-horizons," Automatica, vol. 69, pp. 222-231, 2016

[11] K. Liu, A. Seuret, Y. Xia, F. Gouaisbaut, and Y. Ariba, "Bessel-Laguerre inequality and its application to systems with infinite distributed delays," Automatica, vol. 109, 108562, 2019.
[12] L. Ma, Z. Wang, Q.-L. Han, and Y. Liu, "Dissipative control for nonlinear Markovian jump systems with actuator failures and mixed time-delays," Automatica, vol. 98, pp. 358-362, 2018.

[13] A. Seuret, and F. Gouaisbaut, "Wirtinger-based integral inequality: application to time-delay systems," Automatica, vol. 49, no. 9, pp. 2860 2866, 2013.

[14] A. Seuret, F. Gouaisbaut, and E. Fridman, "Stability of discrete-time systems with time-varying delays via a novel summation inequality," IEEE Trans. Autom. Control, vol. 60, no. 10, pp. 2740-2745, 2015.

[15] A. Seuret, K. Liu, and F. Gouaisbaut, "Generalized reciprocally convex combination lemmas and its application to time-delay systems," Automatica, vol. 95, pp. 488-493, 2018.

[16] L. F. P. Silva, V. J. S. Leite, and E. B. Castelan, "Local stabilization of T-S fuzzy discrete-time systems with time-varying delay in the states and saturating actuators," in Proc. Amer. Control Conf., Wisconsin Center, Milwaukee, USA, 2018, pp. 3615-3620.

[17] L. F. P. Silva, V. J. S. Leite, E. B. Castelan, et al., "Local stabilization of nonlinear discrete-time systems with time-varying delay in the states and saturating actuators," Inf. Sci., vol. 518, pp. 272-285, 2020.

[18] O. Solomon, and E. Fridman, "New stability conditions for systems with distributed delays," Automatica, vol. 49, no. 11, pp. 3467-3475, 2013.

[19] C. de Souza, V. J. S. Leite, L. F. P. Silva, E. B. Castelan, ISS Robust stabilization of state-delayed discrete-time systems with bounded delay variation and saturating actuators, IEEE Trans. Autom. Control, vol. 64, no. 9, pp. 3913-3919, 2019.

[20] S. Tarbouriech, J. M. Gomes da Silva Jr, and G. Garcia, "Delaydependent anti-windup strategy for linear systems with saturating inputs and delayed outputs," Int. J. Robust Nonlin. Control, vol. 14, no. 7, pp. 665-682, 2004.

[21] S. Tarbouriech, G. Garcia, J. M. Gomes da Silva Jr, and I. Queinnec, Stability and stabilization of linear systems with saturating actuators. London, U.K.: Springer-Verlag, 2011.

[22] Z. Wang, Y. Liu, G. Wei, and X. Liu, "A note on control of a class of discrete-time stochastic systems with distributed delays and nonlinear disturbances," Automatica, vol. 46, no. 3, pp. 543-548, 2010.

[23] S. Xu, G. Feng, Y. Zou, J. Huang, "Robust controller design of uncertain discrete time-delay systems with input saturation and disturbances," IEEE Trans. Autom. Control, vol. 57, no. 10, pp. 2604-2609, 2012.

[24] R. Yang, P. Shi, G.-P. Liu, and H. Gao, "Network-based feedback control for systems with mixed delays based on quantization and dropout compensation," Automatica, vol. 47, no. 12, pp. 2805-2809, 2011.

[25] H.-B. Zeng, Y. He, M. Wu, and J. She, "Free-matrix-based integral inequality for stability analysis of systems with time-varying delay," IEEE Trans. Autom. Control, vol. 60, no. 10, pp. 2768-2772, 2015.

[26] C.-K. Zhang, Y. He, L. Jiang, and M. Wu, "An improved summation inequality to discrete-time systems with time-varying delay," Automatica, vol. 74, pp. 10-15, 2016.

[27] C.-K. Zhang, Y. He, L. Jiang, M. Wu, and Q.-G. Wang, "An extended reciprocally convex matrix inequality for stability analysis of systems with time-varying delay," Automatica, vol. 85, pp. 481-485, 2017.

[28] L. Zhang, E.-K. Boukas, and A. Haidar, "Delay-range-dependent control synthesis for time-delay systems with actuator saturation," Automatica, vol. 44, no. 10, pp. 2691-2695, 2008.

[29] X.-M. Zhang, Q.-L. Han, Z. Zeng, "Hierarchical type stability criteria for delayed neural networks via canonical Bessel-Legendre inequalities," IEEE Trans. Cybern., vol. 48, no.5 , pp. 1660-1671, 2018.

[30] B. Zhou, Z. Lin, and G. Duan, "A parametric Lyapunov equation approach to the design of low gain feedback," IEEE Trans. Autom. Control, vol. 53, no. 6, pp. 1548-1554, 2008.

[31] B. Zhou, G. Duan, and Z. Lin, "Global stabilization of the double integrator system with saturation and delay in the input," IEEE Trans. Circuits Syst.-I, vol. 57, no. 6, pp. 1371-1383, 2010.

[32] B. Zhou, "Analysis and design of discrete-time linear systems with nested actuator saturations," Syst. Control Lett., vol. 62, no. 10, pp. 871879, 2013. 